\title{
ARTIGOS
}

Submetido 02.04.2019. Aprovado 24.07.2020

Avaliado pelo sistema double blind review. Editor Científico convidado: Rodrigo Baroni

Versão original | DOI: http://dx.doi.org/10.1590/So034-759020210403

\section{ANÁLISE DOS FATORES QUE CONDICIONAM A ADOÇÃO DE MOBILE HEALTH (MHEALTH)}

\section{An analysis of determinants of the adoption of Mobile Health (mHealth) \\ Un análisis de los factores que condicionan la adopción de Mobile Health (mHealth)}

\author{
Nayra Leandro Miguel Martins¹ | nayra.martins@islagaia.pt | ORCID: 0000-0001-7177-3375 \\ Paulo Duarte² | pduarte@ubi.pt | ORCID: 0000-0001-8449-5474 \\ José Carlos M. R. Pinho3 | jcpinho@eeg.uminho.pt | ORCID: 0000-0003-1829-0299 \\ 1ISLA-Instituto Politécnico de Gestão e Tecnologia, Vila Nova de Gaia, Portugal \\ 2Universidade da Beira Interior, Research Centre in Business Sciences-NECE, Covilhã, Portugal \\ 3Universidade do Minho, Centre for Research in Economics and Management-NIPE, Braga, Portugal
}

\section{RESUMO}

Com a crescente utilização da internet e de tecnologias móveis, este estudo torna-se pertinente uma vez que busca analisar os fatores que condicionam a adoção de Mobile Health ( $m$ Health). Para isso, o modelo conceitual proposto integra a Teoria Unificada de Aceitação e Uso da Tecnologia - Unified Theory of Acceptance and Use of Technology - Utaut2, Condição de Saúde Percebida, e-Literacia em Saúde e Competência de Saúde Percebida como determinantes da adoção do mHealth. Para responder às questões de pesquisa, utilizou-se um questionário on-line aplicado a uma amostra não probabilística de indivíduos brasileiros e portugueses que usaram e não usaram mHealth. Os dados foram analisados por meio dos softwares SPSS e SmartPLS3. Os resultados indicam que a adoção ao mHealth é fortemente impactada apenas por algumas variáveis da Utaut2. Entre os resultados, destaca-se o forte impacto da dimensão da "Expectativa de Desempenho" na adoção ao $m$ Health, tanto entre os utilizadores como entre os não utilizadores.

PALAVRAS-CHAVE | Condição de Saúde Percebida, E-Literacia em Saúde, Competência de Saúde Percebida, $m$ Health, Utaut2.

\section{ABSTRACT}

Given the increasing use of the Internet and mobile technologies, this study is pertinent as it seeks to analyze the factors that determine the adoption of Mobile Health (mHealth). To that end, the proposed conceptual model integrates the Unified Theory of Acceptance and Use of Technology (UTAUT2), Perceived Health Condition, eHealth Literacy and Perceived Health Competence as determinants of the adoption of mHealth. To answer the research questions, we used an online questionnaire administered to a non-probabilistic sample of Brazilian and Portuguese individuals who have or have not used mHealth. Data were analyzed using SPSS and SmartPLS3 software. The results indicate that adoption of mHealth is heavily impacted only by some UTAUT2 variables. The 'Performance Expectancy' dimension was found to heavily impact the adoption of mHealth among both users and non-users.

KEYWORDS I perceived health condition, eHealth literacy, perceived health competence, mHealth, UTAUT2.

\section{RESUMEN}

Con el uso creciente de Internet y las tecnologías móviles, este estudio se vuelve pertinente a medida que busca analizar los factores que determinan la adopción de la Mobile Health (mHealth). Para ello, el modelo conceptual propuesto integra la teoría unificada de aceptación y uso de la tecnología UTAUT2, condición de salud percibida, literacia en e-salud y competencia de salud percibida como determinantes de la adopción de la mHealth. Para responder a las preguntas de investigación, se utilizó un cuestionario online aplicado a una muestra no probabilistica de individuos brasileños y portugueses que usaron y no usaron $m$ Health. Los datos se analizaron utilizando los softwares SPSS y SmartPLS3. Los resultados indican que la adopción de $m$ Health es fuertemente afectada solo por algunas variables de la UTAUT2. Entre los resultados se destacan el fuerte impacto de la dimensión 'expectativa de rendimiento' en la adopción de la mHealth, tanto entre los usuarios como en los no usuarios.

PALABRAS CLAVE I Condición de salud percibida, literacia en e-salud, competencia de salud percibida, mHealth, UTAUT2. 


\section{INTRODUÇÃO}

De acordo com Bernhardt, McClain e Parrott (2004) a prevalência de eHealth (serviços eletrônicos de saúde) está crescendo e vai continuar a crescer por causa das vantagens das ofertas de comunicação de saúde baseadas na internet e, além disso, as empresas obtêm proveitos com a sua implementação. Estas e outras características de comunicação em saúde baseadas na internet podem sugerir porque as pesquisas anteriores indicam o grande potencial da internet para a divulgação de informações de saúde para o público em geral (Cotten \& Gupta, 2004).

De acordo com Dwivedi, Shareef, Simintiras, Lal e Weerakkody (2016), há necessidade de reestruturar o serviço de saúde, mantendo-o coerente e consistente com um estilo de vida dinâmico, flexível e móvel, conectado a uma sociedade dominada pelas Tecnologias de Informação e Comunicação (TIC) e capaz de atrair profissionais da saúde e consultores em TIC para projetar e implementar sistemas profissionais de mHealth.

De acordo com a Organização Mundial de Saúde (OMS), Mobile Health ou mHealth é definido como a prática médica com suporte para dispositivos móveis, tais como: dispositivos para monitoramento de pacientes, smartphones, tablets e outros dispositivos sem fio, incluindo o uso de funções de Short Message Service (SMS), serviços 3G e 4G, Global Positioning System (GPS) e bluetooth. Na literatura científica, o termo mHealth foi definido pela primeira vez por Laxminarayan e Istepanian (2000) como "unwired e-med". Nas duas últimas décadas, o mHealth evoluiu para se tornar um importante canal de comunicação de saúde, para a prestação de cuidados de saúde, a promoção da saúde e o acompanhamento de comportamentos de saúde (Steinhubl, Muse, \& Topol, 2015).

Com a massificação do uso de smartphones, aumentou também a influência de tecnologias móveis na experiência e consumo de serviços de saúde. 0 mHealth torna-se, assim, um mercado que emerge acompanhando a expansão das tecnologias de comunicação móvel (Sweileh et al., 2017). No âmbito do $m$ Health, a literatura sugere que o uso dos aplicativos móveis de saúde serve a diversos objetivos (Riley et al., 2011), nomeadamente melhorar os cuidados de saúde preventivos, limitar o crescimento dos custos com a saúde, reduzir as visitas médicas desnecessárias e melhorar a informação dos utilizadores que passaram a procurar ativamente informações de saúde por meio da internet, utilizando seus dispositivos móveis (Fox \& Duggan, 2012). Para Lee, Hoti, Hughes e Emmerton (2015), os profissionais de saúde deixam, assim, de ser a fonte primária de informação e, dessa forma, o papel dos utilizadores dos serviços de saúde ganha especial relevância.

Explorar a adoção ao mHealth vai ao encontro ao que Kotz, Avancha e Baxi (2009) afirmaram em seu estudo ao dizer que questões sociais, comportamentais e tecnológicas envolvidas na criação de crenças de uma atitude positiva nos consumidores para adotar sistemas $m$ Health são significativamente diferentes dos serviços médicos tradicionais em hospitais, e por isso esse novo contexto deve ser investigado.

Para além dos comportamentos de procura de informação por meio de smartphones, registou-se igualmente um aumento significativo de aplicativos de saúde disponíveis (Fox \& Duggan, 2012; Kratzke \& Cox, 2012), uma vez que o mHealth traz consigo a promessa de resolver os problemas relacionados com a igualdade ao acesso aos cuidados de saúde e também dar uma resposta mais eficaz às novas exigências dos utentes (Duque, Mamede, \& Morgado, 2017). Os aplicativos móveis para a saúde têm, ainda, o potencial de alcançar públicos heterogêneos, como médicos, pacientes, enfermeiros ou mesmo pessoas saudáveis (Free et al., 2010). De acordo com o relatório “mHealth App Developer Economics 2016”, elaborado pela Research2guidance (2016), o mercado de aplicativos $m$ Health é ainda jovem e dinâmico, mas vai continuar a crescer em resultado das vantagens obtidas pelas empresas, que obtêm enormes proveitos com a sua implementação, estimando-se que existam atualmente mais de 350 mil aplicativos disponíveis na Apple Store e Google Play. 
Contudo, o comportamento de adoção de tecnologias de mHealth depende da disponibilidade das pessoas para abraçar a mudança, substituindo hábitos antigos (Shareef, Kumar, \& Kumar, 2014). A literatura existente sugere que a compreensão das motivações cognitivas gerais que desencadeiam o uso de aplicativos de saúde é ainda limitada. A utilização de dispositivos móveis para o cuidado da saúde é ainda um tema deficientemente documentado e explorado, conforme destacado por Cameron, Ramaprasad e Syn (2017).

De acordo com Carlos, Magalhães, Vasconcelos, Silva e Brasil (2016), no Brasil, o uso da tecnologia na assistência à saúde ainda é um desafio. Há a necessidade de aprofundamento dos conhecimentos em saúde, assim como da implementação de iniciativas que busquem um modelo de desenvolvimento socialmente inclusivo e economicamente competitivo (Gadelha \& Costa, 2012).

O relatório da OMS (World Health Organization - WHO, 2019) afirma que as tecnologias digitais oferecem oportunidades concretas para enfrentar os desafios do sistema de saúde, e dessa forma, oferecem o potencial de melhorar a qualidade e a cobertura dos serviços e práticas de saúde. O relatório ainda destaca que intervenções digitais de saúde podem ser usadas, por exemplo, para facilitar a comunicação direcionada aos utentes de modo a ampliar a cobertura de contatos. Tais intervenções também podem ser direcionadas aos profissionais de saúde, para lhes dar acesso mais imediato aos protocolos clínicos por meio de, por exemplo, consultas de telemedicina com outros profissionais de saúde. Há diversas maneiras pelas quais as tecnologias digitais podem ser usadas para atender às necessidades dos sistemas de saúde, e essas tecnologias continuam a evoluir devido à natureza dinâmica do campo.

Entre as vantagens da utilização de mHealth citadas por Carlos et al. (2016), consta a de que os dispositivos móveis podem atender aos usuários tanto na vida cotidiana quanto durante a reabilitação ou internação. Também podem atender aos prestadores de cuidados à saúde durante as visitas de rotina ou de emergência.

De acordo com Biesdorf e Niedermann (2014), a maioria das pessoas recorre aos aplicativos de tecnologias móveis de saúde porque os serviços existentes não atendem às suas necessidades ou a sua qualidade é baixa. Contudo, sem uma compreensão adequada dos fatores motivacionais e cognitivos de adoção e uso dos aplicativos de saúde, torna-se difícil entender completamente o uso desses aplicativos por parte dos indivíduos.

\section{REVISÃO DA LITERATURA}

\section{Condição de Saúde Percebida}

As pesquisas de prevenção e promoção da saúde reconhecem amplamente que as crenças individuais sobre as condições de saúde são preditoras dos comportamentos de saúde (Becker \& Janz, 1984). A saúde percebida, definida por Neter e Brainin (2012) como a saúde autoavaliada pelas pessoas em comparação com outros indivíduos de sua idade e sexo, possui uma forte componente normativa, uma vez que representa para as pessoas uma forma de conscientização da necessidade de adoção de comportamentos. Rai, Chen, Pye e Baird (2013) sugerem que os consumidores que se sentem mais saudáveis são mais abertos a experimentar inovações em saúde. Esses autores baseiam essa expectativa na evidência de que aquelas pessoas com pouca percepção de saúde podem ter fortes relações preexistentes com prestadores de serviços de saúde e estabelecer rotinas terapêuticas que podem resultar em resistência a alternativas de serviço e opções de consumo (Bitner, Brown, \& Meuter, 200o).

De acordo com Shields e Shooshtari (2001), a mudança relativa ao estilo de vida adotado, ao estado físico e aos fatores psicológicos é uma mais-valia para a evolução de uma perspectiva mais otimista da saúde sub- 
jetiva. De um modo geral, quando uma pessoa avalia a sua própria saúde, considera uma série de fatores que muitas vezes não são identificados numa avaliação física (Shields \& Shooshtari, 2001).

\section{Competência de Saúde Percebida}

De acordo com Smith, Wallston e Smith (1995), a Competência de Saúde Percebida representa o grau em que um indivíduo se sente capaz de gerir efetivamente os seus resultados de saúde, bem como a percepção e controle sobre o comportamento e a capacidade de atingir os objetivos. A escala de Competência de Saúde Percebida (Perceived Health Competence Scale - PHCS) foi desenvolvida por Smith et al. (1995) com o intuito de fornecer uma medida de competência percebida em um nível intermediário de especificidade para o qual combina expectativas de resultados e comportamentos.

Bachmann et al. (2016) desenvolveram um estudo com o intuito de avaliar o efeito da Competência de Saúde Percebida na crença de um paciente em sua capacidade de atingir metas relacionadas à saúde. Esses autores afirmaram que pacientes com baixa Competência de Saúde Percebida podem estar em risco de declínio na qualidade de vida relacionada à saúde após a hospitalização e, por isso, são um potencial alvo para aconselhamento e outras intervenções comportamentais.

\section{e-Literacia em Saúde e adoção do mHealth}

e-Literacia em Saúde representa "a capacidade de procurar, encontrar, entender e apreciar informações de saúde de fontes eletrônicas e aplicar o conhecimento adquirido para tratar ou resolver um problema de saúde” (Norman \& Skinner, 2006 b, p. 2). Segundo Bodie e Dutta (2008), um nível de e-Literacia em Saúde elevado não reflete apenas a capacidade para usar a internet para encontrar respostas a questões relacionadas com a saúde, implica também a capacidade de compreender as informações encontradas, avaliar a veracidade delas, discernir a qualidade dos diferentes websites de saúde e usar informação de qualidade para tomar decisões embasadas sobre saúde.

Uma melhor literacia digital de saúde ajuda os pacientes a utilizarem as tecnologias de saúde móvel de modo eficaz. Uma pesquisa mostra que pacientes com menor conhecimento em saúde são menos propensos a adotar a tecnologia mHealth (Mackert, Mabry-Flynn, Champlin, Donovan, \& Pounders, 2016).

A adoção do mHealth tem como principais vantagens a melhoria na troca de dados, a economia de tempo, a redução da perda de informações e, também, na coleta de informações dos utentes em seus ambientes sem necessidade de deslocamento (Anantraman et al., 2002; Barrett, Strayer, \& Schubart, 2004; Koop \& Mösges, 2002), vantagens essas que nem sempre são óbvias para os utilizadores. Com o intuito de melhorar o monitoramento, a prevenção e a detecção de doenças, as diferentes especialidades médicas apostam nas vantagens que os aplicativos e serviços mHealth podem proporcionar. Países em desenvolvimento, onde o acesso aos cuidados de saúde é difícil, procuram aumentar a sua eficácia por meio da integração de serviços mHealth em seus sistemas de saúde (Déglise, Suggs, \& Odermatt, 2012).

\section{Utaut e Utaut2}

Essa teoria foi concebida por Venkatesh, Morris, Davis e Davis (2003) para unificar oito teorias e modelos existentes, a saber: Teoria da Ação Racional (Theory of Reasoned Action - TRA); Modelo de Aceitação de Tecnologia 
(Technology Acceptance Model - TAM); Modelo Motivacional (Motivational Model - MM); Teoria do Comportamento Planejado (Theory of Planned Behavior - TPB); Modelo Combinado TAM e TPB (Combined TAM and TPB - C-TAMTPB); Modelo de Utilização de Computador Pessoal (Model of Personal Computer Utilization - MPCU); Teoria da Difusão da Inovação (Innovation Diffusion Theory - IDT); e Teoria Social Cognitiva (Social Cognitive Theory - SCT). Tais teorias envolvem a temática de como os utilizadores aceitam e utilizam uma tecnologia (Venkatesh \& Morris, 2000; Venkatesh et al., 2003).

Desde sua publicação original, a Utaut serviu como um modelo de referência e foi aplicada numa variedade de estudos sobre tecnologias em ambientes organizacionais e não organizacionais (Dwivedi et al., 2016; Escobar-Rodríguez \& Carvajal-Trujillo, 2014; Tan, 2013). Riffai, Grant e Edgar (2012) constatam que Utaut fornece uma visão refinada de como os determinantes da intenção e do comportamento evoluíram ao longo do tempo. Contudo, há necessidade de uma investigação sistemática e teorização dos fatores salientes que se aplicariam ao uso da tecnologia no contexto do consumidor, nomeadamente no âmbito do mHealth.

Com o objetivo de reforçar a capacidade de explicação da Utaut, Venkatesh, Thong e Xu (2012) desenvolvem a Utaut2, um modelo ainda mais completo em que alteraram algumas das relações existentes na conceituação original da Utaut e introduziram novas ligações e construtos com a intenção de tornar a Utaut2 aplicável no âmbito de um comportamento voluntário, justificando a sua pertinência no âmbito da adoção do $m H$ Health. Assim, no presente estudo, será utilizada a Utaut2, por ser mais recente e se mostrar mais completa. Além disso, é mais coerente com a proposta do trabalho, que engloba o comportamento do consumidor e a utilização da tecnologia de maneira voluntária.

\section{Hipóteses de Pesquisa e Modelo Conceitual}

Tomando por base o modelo Utautz e as dimensões da Condição de Saúde Percebida, Competência de Saúde Percebida e a e-Literacia em Saúde, formulou-se o modelo conceitual expresso na Figura 1, com as hipóteses que a seguir se discriminam com o respectivo suporte teórico.

\section{Condição de Saúde Percebida, e-Literacia em Saúde e adoção ao mHealth}

A Condição de Saúde Percebida não está relacionada apenas com a avaliação da saúde física, mas também com a incidência ou prevalência de morbilidade (que é a taxa de portadores de determinada doença em relação à população total estudada) e mortalidade e, por isso, é um importante instrumento de avaliação da qualidade de saúde e de vida das pessoas (Leal, 2009). Finkelstein (2000), a partir de um estudo com hipertensos, sugere que a percepção que a pessoa tem da sua própria saúde influencia o aumento da busca por cuidados de saúde, ao confirmar que existia uma relação entre o número de visitas ao médico com a Condição de Saúde Percebida.

Diante do exposto e dentro do contexto do atual trabalho, procura-se analisar se os indivíduos com pior autopercepção tendem a adotar com mais facilidade o mHealth e a desenvolver habilidades relacionadas à e-Literacia em Saúde. Desse modo, sugerem-se as seguintes hipóteses:

H1: A Condição de Saúde Percebida influencia positivamente a e-Literacia em Saúde.

H2: A Condição de Saúde Percebida influencia positivamente a adoção ao mHealth. 


\section{Competência de Saúde Percebida e adoção ao mHealth}

Smith et al. (1995) indicam que a Competência de Saúde Percebida é um preditor do comportamento de saúde pretendido e comportamento real. Bachmann et al. (2016) desenvolveram um estudo entre pacientes com doença cardiovascular e concluíram que a Competência de Saúde Percebida prediz o comportamento de saúde e a qualidade de vida relacionada à saúde dos pacientes, bem como a mudança na qualidade de vida relacionada à saúde após a alta. No trabalho de Smith et al. (1995), a percepção de competência em saúde foi relacionada tanto com a intenção quanto com o comportamento real de saúde. Diante do exposto, propõe-se a hipótese:

H3: A Competência de Saúde Percebida influencia positivamente a Adoção ao mHealth.

\section{e-Literacia em Saúde e adoção ao mHealth}

De acordo com Cho, Park e Lee (2014), deve-se considerar que as informações em saúde obtidas por meio dos aplicativos de saúde requerem a competência dos utilizadores em compreender com precisão as informações acessadas, existindo diferenças cognitivas nas habilidades dos indivíduos para encontrar e entender adequadamente a informação em saúde (Basu \& Dutta, 2008; Norman \& Skinner, 2006b). e-Literacia em Saúde está intimamente relacionada à cognição de um indivíduo sobre sua própria capacidade de procurar e entender informações de saúde on-line (Bodie \& Dutta, 2008; Norman \& Skinner, 2006a). Assim, é plausível admitir que uma pessoa com alto nível de e-Literacia em Saúde tenha mais probabilidade de perceber que ela tem uma melhor capacidade para usar aplicativos de saúde, pelo que se propõe a hipótese:

H4: A e-Literacia em Saúde influencia positivamente a adoção ao mHealth.

\section{Utaut2 e adoção de mHealth}

Nesta seção, procedemos a uma breve caracterização de cada uma das subdimensões da Utaut2 e analisamos a respetiva relação com a adoção do mHealth.

A primeira subdimensão em análise refere-se à expectativa de desempenho e é definida como o grau em que um indivíduo acredita que a utilização da tecnologia ajudará a atingir ganhos de desempenho nas suas tarefas (Venkatesh et al., 2012). No contexto do mobile banking, Luo, Li, Zhang e Shim (2010) e Riffai et al. (2012) chegaram à conclusão de que a expectativa de desempenho é um fator-chave para um utilizador aceitar a tecnologia. Consequentemente, admite-se que um utilizador adote o $\mathrm{mHealth}$ pensando nos benefícios que poderá daí obter. Por seu turno, a expectativa de esforço é definida como o grau de facilidade associada com a utilização de um sistema (Venkatesh et al., 2003). Esse construto reflete a percepção de facilidade de uso percebida de um sistema de informação (Kuo \& Yen, 2009; Luarn \& Lin, 2005; Miltgen, Popovič, \& Oliveira, 2013) e tem um impacto positivo sobre a intenção comportamental. Entre os estudos que analisam o impacto da expectativa de esforço na intenção de adotar uma tecnologia, Dwivedi et al. (2016) destacam que a expectativa de esforço tem uma influência positiva na intenção comportamental de adotar mHealth.

Quanto à influência social, é um antecedente direto da intenção comportamental (Oliveira, Faria, Thomas, \& Popovič, 2014). Venkatesh et al. (2003) definem influência social como o grau em que um indivíduo percebe que é importante para os outros acreditar que ele usa a nova tecnologia ou está em conformidade com as expectativas dos outros. As pessoas geralmente ficam desconfortáveis com a incerteza e, por isso, tendem a interagir 
com sua rede social para obterem aval para as suas decisões de adoção, sofrendo, assim, influências sociais informativas e normativas (Burkhardt \& Brass, 1990; Katz \& Tushman, 1979). Contudo, é necessário considerar igualmente as condições facilitadoras de adoção da tecnologia. Estas são definidas como o grau em que um indivíduo acredita que existe uma infraestrutura organizacional e técnica para apoiar a utilização do sistema (Venkatesh et al., 2003). Entre os estudos que analisam o impacto das condições facilitadoras na intenção de adotar uma tecnologia, Dwivedi et al. (2016) destacam que estas têm uma influência positiva na intenção comportamental de adotar $m$ Health. Diante do exposto, pressupõe-se que as condições facilitadoras influenciem de maneira positiva a adoção ao mHealth.

Vários artigos demonstram a influência da motivação hedônica na intenção de usar uma tecnologia e no uso real dessa tecnologia (Heijden, 2004; Thong, Hong, \& Tam, 2006). Especificamente no contexto do consumidor, também foi demonstrado que a motivação hedônica influencia a aceitação e uso da tecnologia (Brown \& Venkatesh, 2005; Childers, Carr, Peck, \& Carson, 2001). Da mesma forma, diversos trabalhos apontam os fatores relacionados ao custo para explicar o comportamento do consumidor (Chong, 2013; Dodds, Monroe, \& Grewal, 1991; Wei, Marthandan, Chong, Ooi, \& Arumugam, 2009). Agarwal, Wang, Xu e Poo (2007), Ong, Poong e Ng (2008) e Xin (2004) mostraram que o custo pode ter relações negativas com as intenções dos consumidores em adotar 0 3G, serviço que pode ser essencial para a adoção de mHealth. Xin (2004), indica que uma das razões pelas quais o serviço de SMS é amplamente utilizado pelas pessoas é devido aos seus baixos custos para permitir que os utilizadores se comuniquem, pelo que se estima que o preço influencie negativamente a adoção ao $\mathrm{mHealth}$.

No que diz respeito ao hábito, este tem sido considerado em muitos estudos como um preditor do uso de uma tecnologia (Kim \& Malhotra, 2005; Kim, Malhotra, \& Narasimhan, 2005; Limayem, Hirt, \& Cheung, 2007). Limayem et al. (2007) demonstraram o efeito direto do hábito sobre o uso continuado de uma tecnologia e um efeito mais moderado do hábito na intenção de uso, pois quanto mais forte é o hábito, menos importante é a intenção consciente de usar a tecnologia (Limayem et al., 2007).

Em face do exposto, este estudo pretende testar se as sete subdimensões relativas à Utaut2 descritas anteriormente influenciam a adoção do mHealth. Para isso, propomos a seguinte hipótese geral e respectivas sub-hipóteses:

H5: As subdimensões da Utaut2 influenciam a adoção do mHealth.

Para validar essa hipótese, procede-se ao teste individual de cada subdimensão da Utaut2, propondo as seguintes sub-hipóteses:

H5a. A expectativa de desempenho influencia positivamente a adoção ao mHealth.

H5b. A expectativa de esforço influencia positivamente a adoção ao mHealth.

H5c. A influência social influencia positivamente a adoção ao mHealth.

$\mathrm{H}_{5}$ d. As condições facilitadoras influenciam positivamente a adoção ao mHealth.

H5e. A motivação hedônica influencia positivamente a adoção ao mHealth.

$\mathrm{H}_{5}$ f. O preço influencia negativamente a adoção ao mHealth.

H5g. O hábito influencia positivamente a adoção ao mHealth. 
A Figura 1 sintetiza graficamente o modelo conceitual, bem como as hipóteses de relações em estudo da presente investigação.

\section{Figura 1. Modelo Inicial de Pesquisa}

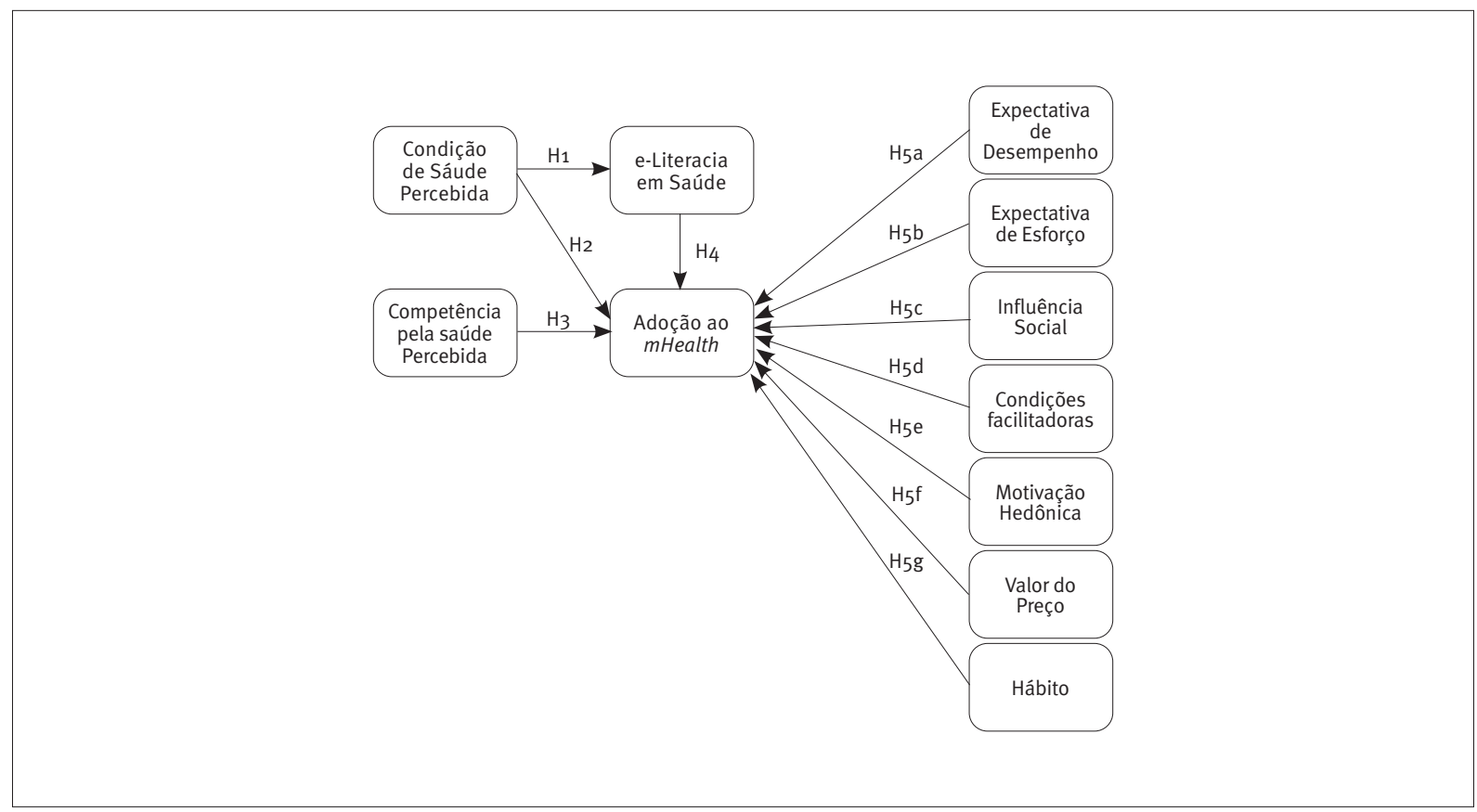

\section{MÉTODO}

\section{Instrumento de coleta de dados e amostra}

O presente estudo adota uma metodologia quantitativa. Nesse sentido, procedeu-se ao desenvolvimento e aplicação de um questionário para obtenção dos dados necessários à avaliação das hipóteses referentes aos fatores que impactam a adoção ao mHealth entre portugueses e brasileiros. Optou-se por comparar essas duas nacionalidades uma vez que, em função de fatores históricos, apresentam muitas semelhanças. Dessa forma, teve-se o intuito de perceber até que ponto essas semelhanças são perceptíveis no que se refere à utilização de uma tecnologia. 0 questionário foi sujeito a um pré-teste com um grupo de estudantes de pós-graduação de nacionalidades portuguesa e brasileira.

0 pré-teste foi realizado de modo a detectar possíveis problemas de compreensão de texto que pudessem enviesar o correto preenchimento. Foram realizados seis pré-testes em Portugal e seis no Brasil. Os respondentes do pré-teste sugeriram alterações na redação de alguns itens; para além dessas alterações, procedeu-se à inclusão de uma pergunta filtro para identificar os respondentes que já haviam experimentado o mHealth (grupo1) e os que nunca haviam experimentado mHealth (grupo 2). Daqui resultaram dois grupos, sendo as análises subsequentes realizadas com base nesses dois grupos. Três respondentes do pré-teste afirmaram que o fato de ter ou não ter experiência com a tecnologia interferia no modo como interpretavam as questões do questionário. 
Dessa forma, após a realização do pré-teste, percebeu-se a necessidade de dividir entre esses dois grupos para conseguir identificar se o inquirido respondia com base em sua experiência de utilização do mHealth ou se respondia com base em uma situação hipotética.

Para a obtenção da amostra, foi usado o método não probabilístico por conveniência, que implicou a escolha dos elementos com base em critérios definidos pelo próprio investigador. Assim, foram enviados convites para um vasto grupo de contatos contendo o link direto para a página web do questionário. 0 questionário esteve on-line por meio da plataforma LimeSurvey entre abril de 2018 e julho de 2018, tendo sido recolhidas 326 respostas válidas. Desse total, 120 dos respondentes já haviam experimentado mHealth e 206 não haviam experimentado mHealth.

\section{Medidas}

A revisão da literatura relevante permitiu identificar os construtos e respetivas escalas de medida que melhor se ajustavam ao problema de pesquisa e que suportaram o desenvolvimento do modelo conceitual proposto (Figura 1). Desse modo, as escalas usadas neste estudo foram baseadas em pesquisas anteriores (e.g. Neter \& Brainin, 2012; Norman \& Skinner, 2006 a; Smith et al., 1995; Tomás, Queirós, \& Ferreira, 2014; Venkatesh et al., 2012), porém alguns indicadores tiveram de ser ajustados ao atual contexto de pesquisa.

Para o construto de e-Literacia em Saúde, foi usada uma versão validada para português por Tomás et al. (2014) da escala original proposta por Norman e Skinner (2006a). Os autores facultarão, caso seja solicitado, as escalas de medida utilizadas no presente estudo. Para avaliação dos itens dos vários construtos, foi usada uma escala de Likert de 7 pontos que varia de (1) discordo totalmente a (7) concordo plenamente.

\section{RESULTADOS}

\section{Caracterização dos respondentes}

Os respondentes foram divididos em dois grupos com base na experiência ou não de mHealth, sendo o grupo 1 composto pelos os respondentes que já haviam experimentado $m$ Health e o grupo 2 pelos que não haviam experimentado mHealth. Da totalidade de elementos do grupo 1, 73,3\% pertencem ao gênero feminino e $26,7 \%$, ao gênero masculino. Por seu turno, no grupo 2, 65,5\% pertencem ao gênero feminino e 34,5\%, ao gênero masculino. Em relação à faixa etária, no grupo 1 há uma preponderância de duas faixas etárias, as que compreendem dos 18 aos 29 anos e dos 30 aos 39 anos, totalizando ambas 82,5\% dos respondentes. Em relação à faixa etária do grupo 2, pode-se observar que também há uma preponderância em duas faixas etárias, que compreendem os indivíduos entre os 18 e 29 anos e entre os 30 e 39 anos, num total de 73,3\%.

Outra informação recolhida diz respeito à nacionalidade dos participantes. Dos respondentes do grupo $1,36,7 \%$ eram portugueses; $62,5 \%$ eram brasileiros; e $0,8 \%$ de outra nacionalidade (belga). No que se refere à nacionalidade dos participantes do grupo 2, há um maior equilíbrio entre os portugueses e brasileiros, 48,5\% e $49 \%$, respectivamente, $2,4 \%$ representando outras nacionalidades (angolana, inglesa e uruguaia).

Relativamente a esse aspecto da nacionalidade, procuramos saber se existem diferenças significativas entre os participantes de nacionalidade portuguesa ou brasileira. Para o efeito, realizaram-se alguns testes de diferença de médias (teste t para amostra independentes), tendo os resultados indicado que não há diferenças estatisticamente significativas entre os respondentes portugueses e brasileiros. 
Na análise efetuada às várias dimensões, verificou-se que a escala de medida relativa Competência de Saúde Percebida (PHCS) apresentava inconsistência, pelo que se optou por efetuar uma análise fatorial exploratória (AFE), com rotação oblíqua em SPSS para avaliar a unidimensionalidade dos diferentes indicadores que pertencem a essa escala. Definiu-se que apenas os fatores que apresentam um eigenvalue superior a > $1 \mathrm{seriam}$ retidos para análise. Dessa análise, extraíram-se dois fatores distintos, tendo o primeiro fator agrupado os itens 1 , 4, 5 e 8; e o segundo fator agrupou os itens 2, 3, 6 e 7. Esses dois fatores explicam 61\% da variância total. 0 KMO (Measure of Sampling Adequacy) é considerado bom $(0,80)$, tal como o respectivo teste de Bartlett de Esfericidade (530,4; $p<0.001)$. Destaca-se que não houve problemas significativos no nível da normalidade, homogeneidade da variância e linearidade. Dada a natureza dos itens, o primeiro fator foi denominado Competência em Saúde e o segundo fator, Ineficiência em Saúde. Após essa análise, optou-se por subdividir o conceito de PHCS nessas duas subdimensões, incluindo, cada uma, quatro itens. Tendo por base essa nova realidade, reformulou-se o modelo inicial, sendo a formulação final apresentada na Figura 2.

\section{Figura 2. Modelo Final de Pesquisa}

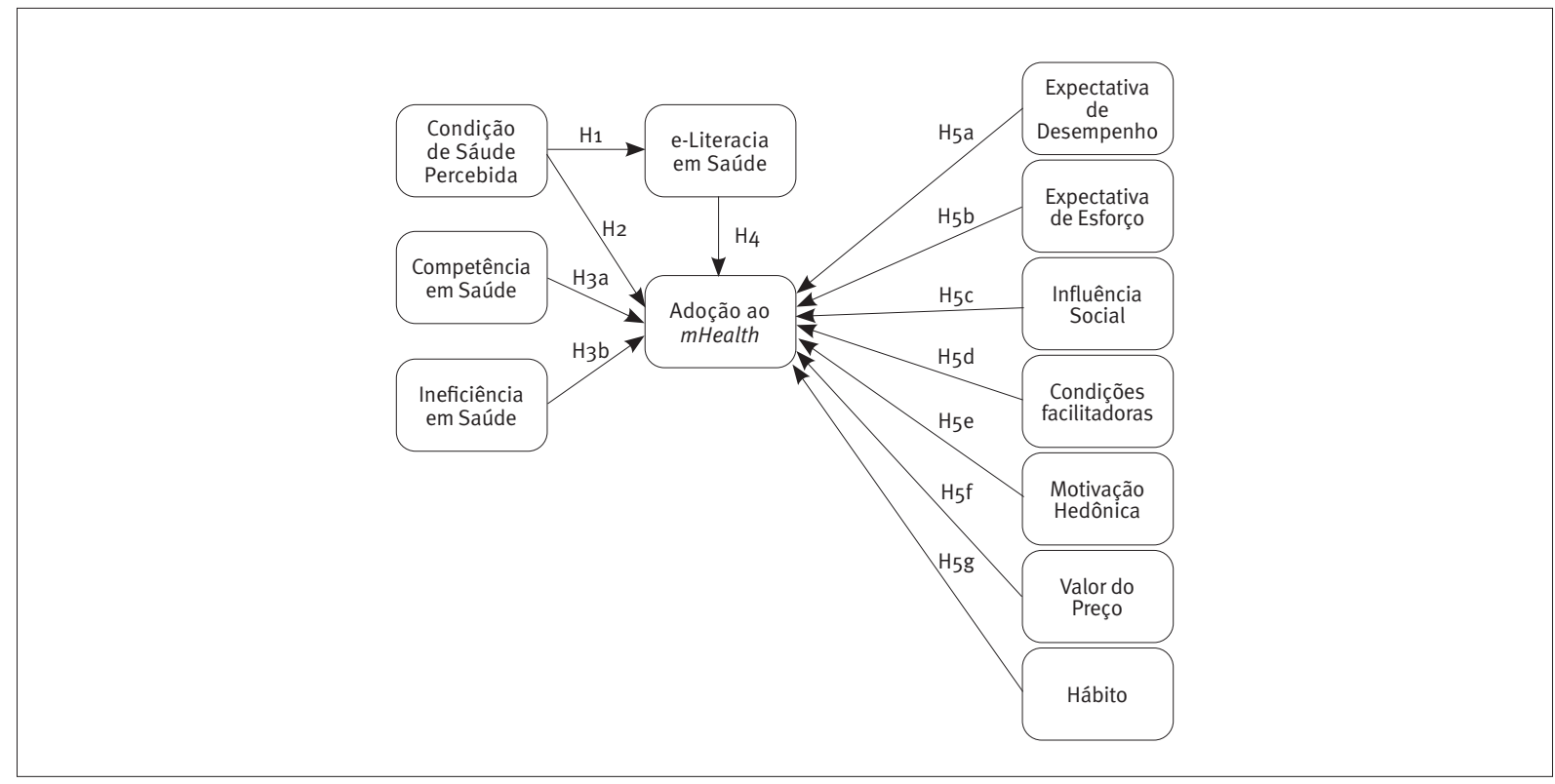

Para testar o modelo exposto na Figura 2, recorreu-se ao método Partial Least Squares (PLS) SmartPLS 3.2.8 (Ringle, Wende, \& Becker, 2015). O PLS funciona muito bem na presença de modelos complexos e é robusto diante de desvios na normalidade dos dados e amostras pequenas (Duarte \& Raposo, 2010). Esse método propõe que a análise seja feita em duas etapas: 1) avaliação do modelo de medida; 2) avaliação do modelo estrutural.

\section{Avaliação do modelo de medida}

No que se refere ao modelo de medida, procedeu-se numa primeira fase à análise de fiabilidade de cada escala de medida para os dois subgrupos em análise (grupo 1: já experimentaram mHealth; grupo 2: não haviam experimentado mHealth). 
Após a análise inicial aos dados do grupo 1, procedeu-se à remoção dos indicadores que apresentam cargas fatoriais ("loading factors") inferiores a 0,60. Em seguida, para assegurarmos a fiabilidade dos diferentes construtos, calculou-se o alfa de Cronbach e Fiabilidade Composta, cujos valores respeitam o limite mínimo de 0,70 (Fornell \& Larcker, 1981) e, como tal, evidenciando um bom nível de consistência interna nas respostas (Bagozzi \& Yi, 1988; Nunnally \& Bernstein, 1994). A mesma conclusão foi estabelecida para o grupo 2. Da mesma forma, a variância média extraída (Average Variance Extracted - AVE) é satisfatória para todos os construtos (acima de 0,50) (Bagozzi \& Yi, 1988) em ambos os grupos, com apenas uma exceção no grupo 2.

A validade discriminante indica o grau de extensão a partir do qual um determinado construto difere dos restantes. Para aferir esse tipo de validade, levamos em conta dois critérios: 1) os indicadores devem apresentar um maior peso no respectivo construto do que nos restantes construtos; 2) a raiz quadrada de AVE, i.e., os valores da diagonal principal da tabela devem ser superiores aos diferentes valores de correlação em linha e em coluna envolvendo o respectivo construto. Conforme se pode constatar por meio das Tabelas 1 e 2, ambos os critérios se cumprem para os dois grupos em análise.

\section{Tabela 1. Correlações e análise discriminante do Grupo 1 (já experimentaram $m$ Health)}

\begin{tabular}{|c|c|c|c|c|c|c|c|c|c|c|c|c|}
\hline Grupo 1 & CFacil & EDesem & EEsforço & Hábito & ISocial & $\begin{array}{c}\text { Ineficiência } \\
\text { em Saúde }\end{array}$ & $\begin{array}{l}\text { Motiv } \\
\text { Hed }\end{array}$ & $\begin{array}{l}\text { Condição } \\
\text { de Saúde }\end{array}$ & $\begin{array}{l}\text { Competência } \\
\text { em Saúde }\end{array}$ & Preço & $\begin{array}{l}\text { Adoção } \\
\text { mHealth }\end{array}$ & e-literacia \\
\hline CFacil & 0.754 & & & & & & & & & & & \\
\hline EDesem & 0.520 & 0.875 & & & & & & & & & & \\
\hline EEsforço & 0.675 & 0.361 & 0.842 & & & & & & & & & \\
\hline Hábito & 0.227 & 0.381 & 0.150 & 0.803 & & & & & & & & \\
\hline ISocial & 0.274 & 0.218 & 0.151 & 0.280 & 0.931 & & & & & & & \\
\hline $\begin{array}{l}\text { Ineficiência } \\
\text { em Saúde }\end{array}$ & 0.161 & -0.064 & 0.206 & -0.074 & -0.107 & 0.782 & & & & & & \\
\hline Motiv_Hed & 0.179 & 0.230 & 0.240 & 0.309 & 0.201 & -0.004 & 0.823 & & & & & \\
\hline $\begin{array}{l}\text { Condição de } \\
\text { Saúde }\end{array}$ & 0.198 & 0.192 & 0.276 & -0.016 & -0.072 & 0.520 & 0.148 & 0.784 & & & & \\
\hline $\begin{array}{l}\text { Competência } \\
\text { em Saúde }\end{array}$ & 0.178 & 0.114 & 0.177 & 0.196 & 0.006 & 0.375 & 0.241 & 0.536 & 0.747 & & & \\
\hline Preço & 0.189 & 0.042 & 0.310 & 0.103 & 0.235 & 0.073 & 0.279 & 0.155 & 0.156 & 0.702 & & \\
\hline $\begin{array}{l}\text { Adoção } \\
\text { mHealth }\end{array}$ & 0.437 & 0.705 & 0.328 & 0.505 & 0.298 & -0.138 & 0.437 & 0.047 & 0.153 & 0.137 & 0.819 & \\
\hline e-literacia & 0.392 & 0.250 & 0.423 & 0.163 & 0.026 & 0.219 & 0.118 & 0.369 & 0.434 & 0.046 & 0.217 & 0.829 \\
\hline
\end{tabular}


Tabela 2. Correlações e análise discriminante do Grupo 2 (não experimentaram mHealth)

\begin{tabular}{|c|c|c|c|c|c|c|c|c|c|c|c|c|}
\hline Grupo 2 & $\begin{array}{l}\text { Cond } \\
\text { Facil }\end{array}$ & $\begin{array}{l}\text { Exp_ }_{-} \\
\text {Esfor }\end{array}$ & $\begin{array}{c}\text { Exp__ }_{-} \\
\text {desemp_ }\end{array}$ & Ineficiencia_ & Infl_soc & $\begin{array}{c}\text { Motiv } \\
\text { Hed }\end{array}$ & PHC & PHCs & Preço & eHealth & hábito & mAdopt \\
\hline Cond_Facil & 0.728 & & & & & & & & & & & \\
\hline Exp_Esfor & 0.457 & 0.914 & & & & & & & & & & \\
\hline $\begin{array}{l}\text { Exp_- } \\
\text { desemp_ }\end{array}$ & 0.415 & 0.305 & 0.929 & & & & & & & & & \\
\hline Ineficiencia_ & 0.066 & 0.114 & -0.061 & 0.740 & & & & & & & & \\
\hline Infl_soc & 0.324 & 0.410 & 0.320 & -0.051 & 0.961 & & & & & & & \\
\hline Motiv_Hed & 0.284 & 0.537 & 0.246 & 0.040 & 0.455 & 0.950 & & & & & & \\
\hline $\mathrm{PHC}$ & 0.095 & 0.070 & 0.055 & 0.461 & -0.071 & 0.019 & 0.809 & & & & & \\
\hline $\mathrm{PHCs}$ & 0.135 & 0.125 & 0.112 & 0.135 & 0.045 & 0.156 & 0.412 & 0.804 & & & & \\
\hline Preço & 0.117 & 0.304 & 0.179 & -0.038 & 0.408 & 0.556 & 0.010 & 0.151 & 0.803 & & & \\
\hline eHealth & 0.251 & 0.300 & 0.109 & 0.109 & 0.021 & 0.106 & 0.165 & 0.132 & 0.046 & 0.831 & & \\
\hline hábito & 0.255 & 0.114 & 0.299 & -0.111 & 0.234 & 0.169 & -0.027 & 0.112 & -0.012 & 0.067 & 0.935 & \\
\hline mAdopt & 0.368 & 0.231 & 0.717 & -0.155 & 0.376 & 0.265 & -0.014 & 0.162 & 0.161 & 0.003 & 0.353 & 0.852 \\
\hline
\end{tabular}

Em face da análise anterior, é possível afirmar que a maioria dos exames psicométricos das diferentes escalas de medida evidencia unidimensionalidade, consistência e robustez conceitual.

\section{Avaliação do modelo estrutural}

Para testar as hipóteses propostas, procedeu-se a uma análise da significância dos coeficientes estruturais, análise dos respetivos testes T e dos valores de $\mathrm{R}^{2}$. Para o efeito, usamos o procedimento bootstrapping (Tabela 3).

Tabela 3. Resultado do Teste de Hipóteses

\begin{tabular}{|c|c|c|c|c|c|c|}
\hline \multirow[b]{2}{*}{ Caminhos } & \multicolumn{3}{|c|}{$\begin{array}{l}\text { Experimentaram mHealth } \\
\text { (grupo 1) }\end{array}$} & \multicolumn{3}{|c|}{$\begin{array}{c}\text { Não experimentaram mHealth } \\
\text { (grupo 2) }\end{array}$} \\
\hline & $\begin{array}{l}\text { Efeitos } \\
\text { Diretos }\end{array}$ & $\begin{array}{c}\text { Valor t } \\
\text { (bootstrap) }\end{array}$ & Suportada & $\begin{array}{l}\text { Efeitos } \\
\text { Diretos }\end{array}$ & $\begin{array}{c}\text { Valor t } \\
\text { (bootstrap). }\end{array}$ & Suportada \\
\hline $\mathrm{H}_{1}: \mathrm{CSP} P$; $;$ e-Literacia & $0.36^{\star \star \star}$ & $5 \cdot 34$ & Sim & $0.16^{*}$ & 2.10 & Sim \\
\hline H2: CSP .... & 0.10 & 1.35 & Não & -0.02 & 0.40 & Não \\
\hline Hза: COMP_S $\ldots . . ;$ ad_mHealth & 0.06 & 0.68 & Não & 0.10 & 1.16 & Não \\
\hline H3b: Inefic_S -...; ad_mHealth & 0.07 & 0.85 & Não & $0.10^{*}$ & 1.65 & Sim \\
\hline H4: e-Literacia_.... & 0.02 & 0.23 & Não & -0.07 & 1.16 & Não \\
\hline 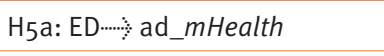 & $0.54^{\star \star \star}$ & 6.56 & $\operatorname{Sim}$ & $0.61^{\star \star *}$ & 11.3 & $\operatorname{Sim}$ \\
\hline $\mathrm{H}_{5} \mathrm{~b}: \mathrm{EE}-\cdots$ ? ad_mHealth & 0.02 & 0.36 & Não & -0.05 & 0.80 & Não \\
\hline $\mathrm{H}_{5 \mathrm{C}}:$ IS $\ldots$; ad_mHealth & 0.04 & 0.61 & Não & $0.13^{*}$ & 1.96 & $\operatorname{Sim}$ \\
\hline $\mathrm{H}_{5} \mathrm{~d}: \mathrm{CF} \cdots \cdots$ ? ad_mHealth & 0.05 & 0.61 & Não & 0.07 & 1.15 & Não \\
\hline $\mathrm{H}_{5} \mathrm{e}: \mathrm{MH} \cdots$ ? ad_mHealth & $0.22^{\star \star \star}$ & $3 \cdot 35$ & $\operatorname{Sim}$ & 0.07 & 0.94 & Não \\
\hline $\mathrm{H}_{5} \mathrm{f}: \mathrm{VP} \ldots . \ldots$ s ad_mHealth & 0.01 & 0.12 & Não & -0.05 & 0.47 & Não \\
\hline H5g: Hab.... $>$ ad_mHealth & $0.18^{*}$ & 2.16 & Sim & $0.09^{*}$ & 1.70 & Sim \\
\hline
\end{tabular}

Baseado em t(1000); * p<0.05 (1.645); **p<0.01(2.327); ***p<0.001(3.092); unilateral.

Legenda: CSP = Condição de Saúde Percebida; e-Literacia=e-Literacia em saúde; ad_mHealth=Adoção ao $m$ Health; COMP_S=Competência em Saúde; Inefic_S= Ineficiência em saúde; $E D$ = Expetativa de desempenho; $E E$ = Expetativa de esforço; IS = Influência social; $C F=$ Condições facilitadoras; $M H$ H = Motivação Hedônica; VP = Valor do Preço; Hab= Hábito. 


\section{Discussão e Considerações Finais}

O foco principal desta pesquisa foi o de propor um modelo que ajude a compreender os antecedentes mais importantes na adoção do mHealth. Para o efeito e de modo a abarcar a diversidade, optou-se por incluir respondentes de duas nacionalidades, tendo observado que não há diferenças estatisticamente significativas associadas a esse fator. Do mesmo modo, considerou-se determinante discriminar se o utilizador experimentou (ou adotou) ou não a plataforma $m$ Health. Para tal, optou-se por dividir os respondentes entre aqueles que já tinham experimentado o mHealth (grupo1) e os que não tinham experimentado essa tecnologia (grupo 2). A análise dos dados foi realizada de maneira separada para esses dois grupos. Em termos gerais, pode-se concluir que, no grupo 1, quatro de 12 hipóteses testadas foram confirmadas, enquanto no grupo 2 foram confirmadas cinco das 12 hipóteses.

Com base nos resultados obtidos, pode-se concluir que, de modo geral, a adoção ao mHealth (AmH) é fortemente impactada por algumas variáveis da Utaut2, embora não o seja por todas. Essa conclusão aplica-se igualmente àqueles respondentes que adotaram previamente o $m$ Health e aos que não adotaram. Relativamente às variáveis em que se verifica um maior impacto, destaca-se a expectativa de desempenho (ED). Esse resultado verifica-se tanto entre os respondentes que já experimentaram mHealth (grupo 1 ) como para aqueles que nunca experimentaram (grupo 2). Esse resultado destaca uma distinção entre facilidade de uso e utilidade. Embora a alta literacia em saúde esteja fortemente correlacionada com a facilidade de uso, o mesmo não acontece com a utilidade e expectativa de desempenho. Apesar de os pacientes conseguirem utilizar as ferramentas de $m H e$ alth, necessitam considerá-las relevantes ou úteis para a melhoria da saúde.

0 mesmo acontece em relação à motivação hedônica e ao hábito. De acordo com o nosso estudo, essas três variáveis (expetativa de desempenho, motivação hedônica e hábito) da Utaut2 assumem um papel importante na adoção do $m$ Health, em particular para os respondentes que já experimentaram essa plataforma (grupo 1). Podemos, nesse sentido, afirmar que, no conjunto, a Hipótese 5 (que agrupa as diferentes subdimensões da Utaut2) seria parcialmente aceita.

Relativamente ao grupo de respondentes que ainda não experimentaram a plataforma mHealth (grupo 2), as variáveis mais relevantes foram a expectativa de desempenho (idêntico ao grupo 1), a influência social e o hábito (idêntico ao grupo 1). À semelhança da análise anterior, poderíamos afirmar que a Hipótese 5 seria também parcialmente aceita.

Para além das variáveis da Utaut2, este estudo foi um pouco mais longe e tentou perceber se haveria alguma influência de um conjunto de variáveis relacionadas com a saúde, como a Condição de Saúde Percebida, a Competência de Saúde Percebida (posteriormente subdividida em competência em saúde e ineficiência em saúde) e a e-Literacia em Saúde na adoção da plataforma mHealth. Dessa análise, foi possível constatar que a Condição de Saúde Percebida tem um impacto significativo em e-Literacia em Saúde, confirmando-se desse modo a Hipótese 1. Esse resultado verifica-se tanto para o primeiro como para o segundo grupo. As restantes hipóteses do grupo 1 ( $\mathrm{H}_{2}$, H3a, H3b e H4) não foram confirmadas pelos dados. Apenas a Hipótese H3b foi confirmada para o grupo 2. Os resultados sugerem, ainda que de maneira tímida, que os desenvolvedores de TICs de saúde devem procurar tornar essas ferramentas mais navegáveis para pacientes com menor nível de e-Literacia em Saúde, uma vez que os utilizadores mais alfabetizados em saúde também apreciam a simplicidade e esta influencia a percepção do desempenho. Assim, uma abordagem de intervenções concebidas para utilizadores menos alfabetizados em saúde, com o foco no design e na usabilidade para usuários com menos experiência em $m$ Health, é benéfica para todos. 
As considerações finais da presente investigação permitem inferir que as novas oportunidades oferecidas pelas novas tecnologias móveis não parecem ter sido totalmente exploradas até o momento. Mapear a evolução do campo permite uma melhor compreensão de seus pontos fortes e fracos, além de informar os passos para o desenvolvimento de pesquisas futuras. Tendo em vista os resultados obtidos, é possível afirmar que o mercado de $m$ Health ainda precisa percorrer um longo caminho para alcançar maior parcela da sociedade. No futuro, os desenvolvedores de TICs na área da saúde e os profissionais do setor devem focar-se mais atentamente nos seus públicos-alvo, garantindo que as tecnologias sejam projetadas para uso real destes.

Persiste, ainda assim, a necessidade de entender como a e-Literacia em Saúde está relacionada à adoção e uso do aplicativos de $m$ Health para garantir que todos os utilizadores consigam ter acesso aos benefícios totais dos avanços tecnológicos, protegendo a privacidade das informações de saúde de modo que estes se envolvam e confiem nas organizações e fornecedores de soluções de $m$ Health, pois, embora a saúde móvel seja frequentemente elogiada como uma ferramenta útil para melhorar o envolvimento do paciente, ela pode não atingir essa meta por si só, sendo necessário utilizar táticas que visem a educação do potencial utilizador para promover um uso significativo do $m$ Health. Tal pode ser conseguido por meio da adoção de estratégias multifacetadas de envolvimento dos utilizadores e pacientes de modo a identificar os conhecimentos e habilidades necessárias para utilizar e compreender os benefícios de gerenciarem a sua própria saúde. Estas conclusões podem ser usadas quer por acadêmicos, governo, decisores políticos, quer por empresas do setor, de modo a maximizar a aceitação e utilização dos serviços mHealth.

\section{Limitações e Estudos Futuros}

Algumas limitações no presente estudo relacionam-se sobretudo com a natureza da amostra e técnica de amostragem, que dificultam a obtenção de dados mais robustos e sobretudo que inviabilizam a generalização das conclusões do presente estudo, apesar do esforço em garantir a diversidade e abrangência dos respondentes. Essas limitações implicam que os resultados sejam analisados com cautela e que sejam restritos às condições de implementação do estudo.

Além disto, como a população do presente trabalho foi escolhida por conveniência, seria interesssante incluir no questionário, por exemplo: a área de conhecimento e profissão dos inquiridos, ou outros fatores que podem expor o participante mais ou menos à tecnologia. Essa sugestão para futura pesquisa torna-se necessária, pois, se parte de um grupo fosse composta principalmente por indivíduos mais propensos a usar um aplicativo, por exemplo, isso poderia influenciar os resultados e diminuir as conclusões.

Nesse sentido, sugere-se também, para futuras pesquisas, o recurso a uma amostra de maior dimensão e probabilística, bem como a utilização dos moderadores (gênero, idade e experiência) no modelo, uma vez que tais moderadores não foram utilizados nesta investigação e podem ser utilizados em pesquisas futuras, dada a relevância demonstrada em estudos anteriores (Venkatesh et al., 2003; Venkatesh et al., 2012).

\section{AGRADECIMENTO}

1. Esta pesquisa foi realizada durante o doutorado financiado pela Coordenação de Aperfeiçoamento de Pessoal de Nivel Superior (Capes) - Ministério da Educação do Brasil, através de uma bolsa de estudos.

2. Apoio do NECE - Research Unit in Business Sciences financiado pelo Multiannual Funding Programme do R\&D Centres da Fundação para a Ciência e a Tecnologia (FCT), número do projeto UIDB/04630/2020. 


\section{REFERÊNCIAS}

Agarwal, N. K., Wang, Z., Xu, Y., \& Poo, D. C. C. (2007). Factors affecting $3 \mathrm{G}$ adoption: An empirical study. PACIS 2007 Proceedings, Paper 3, 256-270. Retrieved from http://aisel. aisnet.org/pacis2007/3

Anantraman, V., Mikkelsen, T., Khilnani, R., Kumar, V. S., Pentland, A., \& Ohno-Machado, L. (2002). Open source handheld-based EMR for paramedics working in rural areas. AMIA - Annual Symposium Proceedings. AMIA Symposium, 12 16. doi: D020002441[pii]

Bachmann, J. M., Goggins, K. M., Nwosu, S. K., Schildcrout, J. S., Kripalani, S., \& Wallston, K. A. (2016). Perceived health competence predicts health behavior and health-related quality of life in patients with cardiovascular disease. Patient Education and Counseling, 99, 2071-2079. doi: 10.1016/j. pec.2016.07.020

Bagozzi, R. P., \& Yi, Y. (1988). On the evaluation of structural equation models. Journal of the Academy of Marketing Science, 16(1), 74-94. doi: 10.1007/bfo2723327

Barrett, J. R., Strayer, S. M., \& Schubart, J. R. (2004). Assessing medical residents' usage and perceived needs for personal digital assistants. International Journal of Medical Informatics, 73(1), 25-34. doi: 10.1016/j.ijmedinf.2003.12.005

Basu, A., \& Dutta, M. J. (2008). The relationship between health information seeking and community participation: The roles of health information orientation and efficacy. Health Communication, 23(1), 70-79. doi: $10.1080 / 10410230701807121$

Becker, M. H., \& Janz, N. K. (1984). The health belief model: A decade later. Health Education Quarterly, 11(1), 1-47. doi: 10.1177/109019818401100101

Bernhardt, J. M., McClain, J., \& Parrott, R. L. (2004). Online health communication about human genetics: Perceptions and preferences of internet users. CyberPsychology and Behavior, 7(6), 728-733. doi: $10.1089 /$ cpb.2004.7.728

Biesdorf, S., \& Niedermann, F. (2014). Healthcare’s digital future. McKinsey \& Company.

Bitner, M. J., Brown, S. W., \& Meuter, M. L. (2000). Technology infusion in service encounters. Journal of the Academy of Marketing Science, 28(1), 138-149. doi: 10.1177/0092070300281013

Bodie, G. D., \& Dutta, M. J. (2008). Understanding health literacy for strategic health marketing: eHealth literacy, health disparities, and the digital divide. Health Marketing Quarterly, 25(1-2), 175-203. doi: 10.1080/07359680802126301

Brown, S. A., \& Venkatesh, V. (2005). Model of adoption and technology in households: A baseline model test and extension incorporating household life cycle. MIS Ouarterly, 29(3), 399-426. doi: 10.2307/25148690

Burkhardt, M. E., \& Brass, D. J. (1990). Changing patterns or patterns of change: The effects of a change in technology on social network structure and power. Administrative Science Quarterly, 35(1), 104-127. doi: 10.2307/2393552
Cameron, J. D., Ramaprasad, A., \& Syn, T. (2017). An ontology of and roadmap for mHealth research. International Journal of Medical Informatics, 100, 16-25. doi: 10.1016/j. ijmedinf.2017.01.007

Carlos, D. A. O., Magalhães, T. O., Vasconcelos, J. E., Filho, Silva, R. M., \& Brasil, C. C. P. (2016, setembro). Concepção e avaliação de tecnologia mHealth para promoção da saúde vocal. Risti Revista Ibérica de Sistemas e Tecnologias de Informação, (19), 46-6o. doi: 10.17013/risti.19.46-60

Childers, T. L., Carr, C. L., Peck, J., \& Carson, S. (2001). Hedonic and utilitrian motivations for online retail shopping behavior. Journal of Retailing, 77, 511-535. doi: 10.1016/So0224359(01)00056-2

Cho, J., Park, D., \& Lee, H. E. (2014). Cognitive factors of using health apps: Systematic analysis of relationships among health consciousness, health information orientation, eHealth literacy, and health app use efficacy. Journal of Medical Internet Research, 16(5), e125. doi: 10.2196/jmir.3283

Chong, A. Y. L. (2013). A two-staged SEM-neural network approach for understanding and predicting the determinants of m-commerce adoption. Expert Systems with Applications, 40(4), 1240-1247. doi: 10.1016/j.eswa.2012.08.067

Cotten, S. R., \& Gupta, S. S. (2004). Characteristics of online and offline health information seekers and factors that discriminate between them. Social Science and Medicine, 59(9), 1795-1806. doi: 10.1016/j.socscimed.2004.02.020

Déglise, C., Suggs, L. S., \& Odermatt, P. (2012). Short Message Service (SMS) applications for disease prevention in developing countries. Journal of Medical Internet Research, 14(1), e3. doi: 10.2196/jmir.1823

Dodds, W. B., Monroe, K. B., \& Grewal, D. (1991). Effects of price, brand, and store information on buyers' product evaluations. Journal of Marketing Research, 28(3), 307-319. doi: $10.2307 / 3172866$

Duarte, P. A. O., \& Raposo, M. L. B. (2010). A PLS model to study brand preference: An application to the mobile phone market. In Handbook of partial least squares, 449-485. Springer, Berlin, Heidelberg. doi: 10.1007/978-3-540-32827-8_21

Duque, C., Mamede, J., \& Morgado, L. (2017). Iniciativas de mHealth em Portugal. In: CISTI 2017: 12th Iberian Conference on Information Systems and Technologies. 1-6, IEEE. doi: 10.23919/CISTI.2017.7975803

Dwivedi, Y. K., Shareef, M. A., Simintiras, A. C., Lal, B., \& Weerakkody, V. (2016). A generalised adoption model for services: A cross-country comparison of mobile health (m-health). Government Information Quarterly, 33(1), 174-187. doi: 10.1016/j.giq.2015.06.003

Escobar-Rodríguez, T., \& Carvajal-Trujillo, E. (2014). Online purchasing tickets for low cost carriers: An application of the unified theory of acceptance and use of technology (Utaut) model. Tourism Management, 43, 70-88. doi: 10.1016/j. tourman.2014.01.017 
Finkelstein, M. M. (2000). Hypertension, self-perceived health status and use of primary care services. Canadian Medical Association Journal, 162(1), 45-46. Retrieved from: https:// www.cmaj.ca/content/162/1/45/tab-article-info

Fornell, C., \& Larcker, D. F. (1981). Structural equation models with unobservable variables and measurement error: Algebra and statistics. Journal of Marketing Research, 18(3), 382. doi: $10.2307 / 3150980$

Fox, S., \& Duggan, M. (2012). Mobile Health 2012. Pew Research Center's Internet \& American Life Project. Retrieved from http://www.pewinternet.org/2012/11/o8/mobilehealth-2012/

Free, C., Phillips, G., Felix, L., Galli, L., Patel, V., \& Edwards, P. (2010). The effectiveness of M-health technologies for improving health and health services: a systematic review protocol. BMC Research Notes, 3, 250. doi: 10.1186/1756 0500-3-250

Gadelha, C. A. G., \& Costa, L. S. (2012). Saúde e desenvolvimento no Brasil: Avanços e desafios. Revista de Saúde Pública, 46(1), 13-20. doi: 10.1590/s0034-89102012005000062

Heijden, H. Van der. (2004). User acceptance of hedonic information systems. MIS Quarterly, 28(4), 695-704. doi: $10.2307 / 25148660$

Katz, R., \& Tushman, M. (1979). Communication patterns, project performance, and task characteristics: An empirical evaluation and integration in an R\&D setting. Organizational Behavior and Human Performance, 23(2), 139-162. doi: 10.1016/0030-5073(79)90053-9

Kim, S. S., \& Malhotra, N. K. (2005). A longitudinal model of continued IS use: An integrative view of four mechanisms underlying postadoption phenomena. Management Science, 51(5), 741-755. doi: 10.1287/mnsc.1040.0326

Kim, S. S., Malhotra, N. K., \& Narasimhan, S. (2005). Research note - Two competing perspectives on automatic use: A theoretical and empirical comparison. Information Systems Research, 16(4), 418-432. doi: 10.1287/isre.1050.0070

Koop, A., \& Mösges, R. (2002). The use of handheld computers in clinical trials. Controlled Clinical Trials, 23(5), 469-480. doi: 10.1016/S0197-2456(02)00224-6

Kotz, D., Avancha, S., \& Baxi, A. (2009). A privacy framework for mobile health and home-care systems. Proceedings of the First ACM Workshop on Security and Privacy in Medical and Home-Care Systems - Spimacs '09, November 1, 1-12. doi: 10.1145/1655084.1655086

Kratzke, C., \& Cox, C. (2012). Smartphone technology and apps: Rapidly changing health promotion. International Electronic Journal of Health Education, 15, 72-82. doi: ISSN-1529-1944

Kuo, Y. F., \& Yen, S. N. (2009). Towards an understanding of the behavioral intention to use $3 \mathrm{G}$ mobile value-added services. Computers in Human Behavior, 25(1), 103-110. doi: 10.1016/j. chb.2008.07.007

Laxminarayan, S., \& Istepanian, R. S. H. (2000). Unwired E-MED: The next generation of wireless and Internet telemedicine systems. IEEE Transactions on Information Technology in Biomedicine, 4(3), 189-193. doi: 10.1109/TITB.2000.5956074
Leal, S. A. (2009). Estado de saúde auto-percebido: Índice de massa corporal e percepção da imagem corporal em utentes dos cuidados de saúde primários (Dissertação de mestrado, Faculdade de Psicologia e Ciências de Educação , Universidade de Lisboa, Lisboa, Portugal).

Lee, K., Hoti, K., Hughes, J. D., \& Emmerton, L. M. (2015). Consumer use of "Dr Google": A survey on health informationseeking behaviors and navigational needs. Journal of Medical Internet Research, 17(12), e288. doi: 10.2196/jmir.4345

Limayem, M., Hirt, S. G., \& Cheung, C. M. (2007). How habit limits the predictive power of intention: The case of information systems continuance. MIS Quarterly, 31(4), 705-737. doi: $10.2307 / 25148817$

Luarn, P., \& Lin, H.-H. (2005). Toward an understanding of the behavioral intention to use mobile banking. Computers in Human Behavior, 21(6), 873-891. doi: 10.1016/j. chb.2004.03.003

Luo, X., Li, H., Zhang, J., \& Shim, J. P. (2010). Examining multidimensional trust and multi-faceted risk in initial acceptance of emerging technologies: An empirical study of mobile banking services. Decision Support Systems, 49(2), 222-234. doi: $10.1016 /$ j.dss.2010.02.008

Mackert, M., Mabry-Flynn, A., Champlin, S., Donovan, E. E., \& Pounders, K. (2016). Health literacy and health information technology adoption: The potential for a new digital divide. Journal of Medical Internet Research, 18(10), e264. doi: 10.2196/jmir.6349

Miltgen, C. L., Popovič, A., \& Oliveira, T. (2013). Determinants of end-user acceptance of biometrics: Integrating the "big 3" of technology acceptance with privacy context. Decision Support Systems, 56, 103-114. doi: 10.1016/j.dss.2013.05.010

Neter, E., \& Brainin, E. (2012). eHealth literacy: Extending the digital divide to the realm of health information. Journal of Medical Internet Research, 14(1), e19. doi: 10.2196/jmir.1619

Norman, C. D., \& Skinner, H. A. (2006a). eHEALS: The eHealth literacy scale. Journal of Medical Internet Research, 8(4), e27. doi: 10.1525/cmr.2014.57.1.67

Norman, C. D., \& Skinner, H. A. (2006b). eHealth literacy: Essential skills for consumer health in a networked world. Journal of Medical Internet Research, 8(2), 1-11. doi: 10.2196/ jmir.8.2.e9

Nunnally, J., \& Bernstein, I. (1994). Psychometric theory (3rd ed.). New York, USA: McGraw-Hill.

Oliveira, T., Faria, M., Thomas, M. A., \& Popovič, A. (2014). Extending the understanding of mobile banking adoption: When Utaut meets TTF and ITM. International Journal of Information Management, 34(5), 689-703. doi: 10.1016/j. ijinfomgt.2014.06.004

Ong, J. W., Poong, Y. S., \& Ng, T. H. (2008). 3G services adoption among university students: Diffusion of innovation theory. Communications of the IBIMA, 3(16), 114-121.

Rai, A., Chen, L., Pye, J., \& Baird, A. (2013). Understanding determinants of consumer mobile health usage intentions, assimilation, and channel preferences. Journal of Medical Internet Research, 15(8), e149. doi: 10.2196/jmir.2635 
Research2guidance. (2016). mHealth App Developer Economics 2016. Retrieved from https://research2guidance.com/r2g/ r2g-mHealth-App-Developer-Economics-2016.pdf

Riffai, M. M. M. A., Grant, K., \& Edgar, D. (2012). Big TAM in Oman: Exploring the promise of on-line banking, its adoption by customers and the challenges of banking in Oman. International Journal of Information Management, 32(3), 239250. doi: 10.1016/j.ijinfomgt.2011.11.007

Riley, W. T., Rivera, D. E., Atienza, A. A., Nilsen, W., Allison, S. M., \& Mermelstein, R. (2011). Health behavior models in the age of mobile interventions: Are our theories up to the task? Translational Behavioral Medicine, 1(1), 53-71. doi: 10.1007/ S13142-011-0021-7

Ringle, C. M., Wende, S., \& Becker, J. M. (2015). SmartPLS 3: SmartPLS GmbH, Boenningstedt. Journal of Service Science and Management, 10(3). Retrivied from: https://www.scirp. org/journal/JSSM/

Shareef, M. A., Kumar, V., \& Kumar, U. (2014). Predicting mobile health adoption behaviour: A demand side perspective. Journal of Customer Behaviour, 13(3), 187-205. doi: 10.1362/1 $47539214 X_{14103453768697}$

Shields, M., \& Shooshtari, S. (2001). Determinants of selfperceived health. Health Reports - Statistics Canada, 13(1), 35-52. Retrieved from: https://pubmed.ncbi.nlm.nih. gov/15069807

Smith, M. S., Wallston, K. A., \& Smith, C. A. (1995). The development and validation of the perceived health competence scale. Health Education Research, 10(1), 51-64. doi: 10.1093/her/10.1.51

Steinhubl, S. R., Muse, E. D., \& Topol, E. J. (2015). The emerging field of mobile health. Science Translational Medicine, 7(283), 283rv3-283rv3. doi: 10.1126/scitranslmed.aaa3487

Sweileh, W. M., Al-Jabi, S. W., AbuTaha, A. S., Zyoud, S. H., Anayah, F. M. A., \& Sawalha, A. F. (2017). Bibliometric analysis of worldwide scientific literature in mobile - health: 2006 2016. BMC Medical Informatics and Decision Making, 17 (1), 72. doi: 10.1186/s12911-017-0476-7
Tan, P. J. B. (2013). Applying the Utaut to understand factors affecting the use of english e-learning websites in Taiwan. SAGE Open, 3(4), 1-12. doi: 10.1177/2158244013503837

Thong, J. Y., Hong, S. J., \& Tam, K. Y. (2006). The effects of post adoption beliefs on the expectation-confirmation model for information technology continuance. International Journal of Human Computer Studies, 64(9), 799-810. doi: 10.1016/j. ijhcs.2006.05.001

Tomás, C. C., Queirós, P. J. P., \& Ferreira, T. J. R. (2014). Análise das propriedades psicométricas da versão portuguesa de um instrumento de avaliação de e-Literacia em saúde. Revista de Enfermagem Referência, série IV(2), 19-28. Retrieved from http://www.scielo.gpeari.mctes.pt/scielo.php?script=sci_ arttext\&pid=S0874-02832014000200003\&lang=pt. doi: $10.12707 /$ riv14004

Venkatesh, V., \& Morris, G. M. (2000). Why don't men ever stop to ask for direction? Gender, social influence and their role in technology acceptance and usage behaviour. MIS Quarterly, 24(1), 115-139. doi: 10.2307/3250981

Venkatesh, V., Morris, M. G., Davis, G. B., \& Davis, F. D. (2003). User acceptance of information technology: Toward a unified view. MIS Quarterly, 27(3), 425-478. doi: 10.2307/30036540

Venkatesh, V., Thong, J. Y. L., \& Xu, X. (2012). Consumer acceptance and use of information technology: Extending the unified theory of acceptance and use of technology. MIS Quarterly, 36(1), 157-178. doi: 10.2307/41410412

Wei, T. T., Marthandan, G., Chong, A. Y. L., Ooi, K. B., \& Arumugam, S. (2009). What drives Malaysian m-commerce adoption? An empirical analysis. Industrial Management \& Data Systems, 109(3), 370-388. doi: 10.1108/02635570910939399

World Health Organization. (2019). WHO guideline: Recommendations on digital interventions for health system strengthening. Geneva, Switzerland. Licence: CC BY-NC-SA 3.0 IGO.

Xin, X. (2004). A model of $3 \mathrm{G}$ adoption. AMCIS 2004 Proceedings. Paper 329, 2755-2762. Retrieved from http://aisel.aisnet.org/ amcis2004/329

\section{CONTRIBUIÇÃO DOS AUTORES}

Nayra Martins, Paulo Duarte e José Carlos Pinho trabalharam na conceitualização e abordagem teóricametodológica. A revisão teórica foi conduzida pela Nayra Martins, Paulo Duarte e José Carlos Pinho. A coleta de dados foi coordenada pelo(a) Nayra Martins, Paulo Duarte e José Carlos Pinho. Participaram da análise de dados Nayra Martins, Paulo Duarte e José Carlos Pinho. Todos os autores participaram da redação e revisão final do manuscrito. 